\title{
Prediction of Setting for Admixture Modified Mortars using the VCCTL
}

\author{
Benjamin E. Watts \\ University of Florida, Engineering School of Sustainable Infrastructure and the Environment, \\ 365 Weil Hall, Gainesville FL \\ Christopher. C. Ferraro (Corresponding Author) \\ University of Florida, Engineering School of Sustainable Infrastructure and the Environment, \\ 365 Weil Hall, Gainesville FL
}

\begin{abstract}
The time at which portland cement concrete transitions from a viscous suspension of particles to a rigid interconnected matrix is commonly referred to as "time of set" and is a critical parameter when placing concrete structures. Use of chemical admixtures in concrete for water reduction or acceleration of hydration alters setting behavior; laboratory quality control testing is required to define the altered setting behavior. Use of the Virtual Cement and Concrete Testing Laboratory (VCCTL) for prediction of early age concrete behavior has been limited to plain portland cement concrete. Direct modeling of kinetic and thermodynamic behavior for admixture modified concrete remains challenging. An indirect method to simulate admixture alteration of set is proposed. Hydration simulations are time calibrated using heat of hydration curves obtained from admixture bearing pastes. Simulation results are compared to mortar setting tests, with agreement in certain cases falling within standard specified precision values.
\end{abstract}

Keywords: Admixtures, Setting, VCCTL, Isothermal Calorimetry, Hydration 


\section{Background}

The early age hydration of Portland cement is characterized by dissolution and reprecipitation of cement phases and hydration products. The primary early age contributor to strength development, tricalcium silicate $\left(\mathrm{C}_{3} \mathrm{~S}\right)$, dissolves and combines with water to form calcium-silicate-hydrate (C-S-H) and portlandite or calcium hydroxide $(\mathrm{CH})$ [1]. These hydration products precipitate on the surface of cement particles and grow outward. As this growth progresses, the particles connect and become rigidly bonded together [2]. This process occurs on a continuous basis; however, the primary practical concern is the limit of workability, or the time at which the mixture is no longer plastic and cannot be properly finished [1]. This is commonly referred to as initial set.

The time required for the cement particles in suspension to become interconnected is a function of the physical characteristics of the suspension and the rate at which the hydration reaction progresses. The water to cement ratio $(\mathrm{w} / \mathrm{c})$ has the greatest effect; as the amount of water is reduced, the distance between particles and the time required for them to become interconnected declines [3]. The size of the particles also influences the rate at which dissolutionreprecipitation occurs; a finer average particle size increases the available surface area for reaction to occur, thus, accelerating set [1]. The particles in unmodified portland cement paste also have a tendency to flocculate into clumps, which can reduce the free water in suspension and contribute to faster setting [4]. Flocculation is driven by weak and reversible electrostatic attraction between the cement particles; most water reducing admixtures operate by negating this attraction, with a resultant increase in free water [1].

The setting of a portland cement is chemically regulated by the addition of calcium sulfate as gypsum. The proper amount of gypsum delays the otherwise extremely rapid hydration of tricalcium aluminate $\left(\mathrm{C}_{3} \mathrm{~A}\right)$, consequently, preventing immediate stiffening and providing a period of workability [5]. An overdose of sulfate can result in a different phenomenon, known as "false set" in cements with little or no $\mathrm{C}_{3} \mathrm{~A}$, in which the mixture appears to set, but can be made fluid again with sufficient mixing [4].

Setting is also influenced by any environmental or chemical conditions which regulate hydration in general. Hot or cold temperatures accelerate or retard hydration, and in turn 
accelerate or delay set, respectively. The presence of certain types of admixtures can also accelerate or retard set, either as a direct result of their method of action, or as a side effect of their primary function.

\subsection{Chemical Admixtures}

Chemical admixtures are commonly used in cement-based mixtures to reduce water demand, increase working time, or accelerate strength development. These additions frequently modify the hydration behavior of portland cement, and can impact the setting time of the mixture. Water reducers induce an electrostatic or stearic repulsion between cement particles by coating them with negatively charged long chain polymers [6]. This disperses the particles evenly in solution, effectively increasing the free water and reducing the suspension viscosity [2]. Because these polymers are adsorbed onto the surfaces of cement particles, they limit hydration as long as they are active. This effect, combined with the increased distance between the particles due to dispersion, results in an increase in the time required for set to occur. Due to this phenomenon, many water reducing admixtures are also classified as retarding admixtures even though other methods of set retardation exist.

Water reducers can be loosely grouped into two categories based on their active chemical species. Most normal water reducing admixtures are based on lignosulfonic acid salts, which are derived from the wood pulp production process; higher molecular weight species tend to produce a more pronounced water reducing effect [6]. The purity of a lignosulfonate-based admixture can influence its retarding effect, as can the type of salt it is based on. Less refined compounds retain crude sugars that can act as retarders, while sodium lignosulfonates typically retard less than calcium lignosulfonates [7]. Normal water reducing admixtures are typically lignosulfonate based formulations; a more substantial water reducing effect typically requires a different class of chemicals. For a water reducing effect more dramatic than that obtained via lignosuflonate compounds, polycarboxylate or polyacrylate-based formulations can be employed, though they can also be used as normal water reducing admixtures. This group of water reducers is based on a polyacrylate or polycarboxylate backbone with grafted polyether molecular chains [8]. This type of admixture can have a broad range of water reducing effects based on the specific characteristics of the backbone molecule as well as the grafted chains [7,9] 
Retarding admixtures operate by reducing the solubility of the calcium silicate phases, typically by coating particles in a barrier that hinders the dissolution of calcium ions [6]. This coating can have a water reducing effect as well, but classes of admixtures that do not alter the workability of the mix exist, notably those based on soluble phosphates. In addition to a set retarding effect, the use of retarding admixtures in mass concrete structures can prevent thermal cracking by reducing the rate of heat generation and the corresponding differential thermal stresses [2].

Accelerating admixtures based on soluble inorganic salts operate by shortening the induction period and accelerating the hydration of silicates [8], increasing the rate at which C-S$\mathrm{H}$ forms [10], quickening set and hydration in general. A historically common and effective accelerator is calcium chloride, though alternatives such as calcium nitrite are used in reinforced concrete systems to eliminate corrosion caused by chloride ions in close proximity to steel. Calcium nitrite admixtures are frequently marketed as both accelerating and corrosion inhibiting admixtures for this reason.

\subsection{Portland Cement Hydration Kinetics}

An intrinsic property of Portland cement hydration is that it is exothermic and irreversible [4]. A typical Portland cement heat evolution curve exhibits an immediate sharp spike in heat release, followed by a period of reduced heat generation. This period of low heat evolution is referred to as the "induction period" and corresponds to the time period prior to initial setting. The end of the induction period is marked by a steady rise in heat generation, corresponding to the hydration of $\mathrm{C}_{3} \mathrm{~S}$ and $\mathrm{C}_{2} \mathrm{~S}$. This reaction peaks approximately 10 hours after the reaction starts for unmodified portland cement pastes [2]. The rate of heat release then begins to decline as the quantity of available $\mathrm{C}_{3} \mathrm{~S}$ diminishes and densification of the microstructure begins to limit the diffusion of ions.

Isothermal conduction calorimetry is used to measure the heat release over time of cementitious mixtures. Two vials of equal thermal mass, one containing an inert reference material and one containing the specimen being measured, are sealed in an insulated and constant temperature environment. As the reaction proceeds, the heat flow into the surroundings from both the specimen and reference are measured. The difference between the heat flow measurements of the specimen and reference represent the heat generated by the reaction. The 
inert reference vial allows for slight swings in environmental temperature to be compensated for; because the reference and specimen have equal thermal masses, any changes in environmental temperature have the same effect on both vials [11].

Chemical admixtures modify the kinetics of cement hydration based on their type and dosage rate, typically by altering the duration and location of the heat release peak associated with the hydration of $\mathrm{C}_{3} \mathrm{~S}$ at the end of the induction period [2]. While a relationship between the heat evolution behavior and setting time of Portland cement is probable, direct predictions of set behavior are difficult considering a heat evolution curve provides no phase specific information or characteristics of the evolving microstructure.

\subsection{Hydration Modeling and the VCCTL}

The Virtual Cement and Concrete Testing Laboratory software provides the capability to create and hydrate virtual cementitious materials, which are constructed in software using characteristic data from the real materials they are intended to represent. Volume fraction, surface area fraction and two dimensional spatial distribution data for the four primary cement phases $\left(\mathrm{C}_{3} \mathrm{~S}, \mathrm{C}_{2} \mathrm{~S}, \mathrm{C}_{3} \mathrm{~A}\right.$, and $\left.\mathrm{C}_{4} \mathrm{AF}\right)$ are obtained via scanning electron microscopy (SEM) combined with energy dispersive $\mathrm{x}$-ray spectroscopy (EDS). These imaging techniques are used to create a false color image of polished section of the raw cement of interest. The volume and surface area fractions are measured from this image, along with a two point spatial distribution function for each of the calcium silicate phases. These data are combined with bulk particle size distribution and mass fractions for the different sulfate phases to create a three dimensional virtual microstructure, which is representative of the initial, pre-hydration cement paste $[12,13]$.

Modern Portland cements are permitted to contain up to $5 \%$ limestone by mass per ASTM C150 [14]. The role of limestone in Portland cement has been historically believed to be an inert filler [15] allowing the total amount of portland cement to be increased without additional energy cost. Modern research has found that limestone can exhibit limited reactivity in portland cement, resulting in modified hydration behavior. This can influence setting time, as limestone fines provide nucleation sites for hydration products [16]. The VCCTL provides provisions for incorporation of limestone into the initial microstructure, and accounts for the additional nucleation sites provided, as well as, the limited chemical reactivity of the limestone itself. Experimental verification of this capability of the VCCTL found that the simulations 
agreed with degree of hydration data well for different replacement percentages and water to cementitious material ratios [17].

In addition to the fundamental parameters of the mixture, such as the particle size distribution, water to binder ratio, and composition of the cement, several options exist to enable the microstructure to more closely match the reality of the system being simulated. The particles in the microstructure can be dispersed to different degrees to mimic the effects of water reducing admixtures $[18,19]$. By default, the particles generated are spherical; however, realistic particle shapes can be used to create a more accurate analogue for real cementitious systems and have a pronounced effect on the early age results of the VCCTL [20]. The size of the microstructure is also user defined, with each three dimensional pixel (or voxel) representing a cubic micrometer of space. The default size of the virtual microstructure is a cube 100 micrometers on each side.

A number of options to define the specifics of the hydration conditions of the simulation are also provided. The initial temperature is input, and thermal state of the evolving microstructure can be defined, either as isothermal, semi-adiabatic (with a fixed heat transfer rate out of the microstructure), or adiabatic. Curing conditions can be simulated, with options to either provide additional water for reaction as long as it can diffuse through the microstructure (saturated) or to limit the water to that initially present (sealed). There is also the option to specify the deactivation percentage and duration for the surfaces of specific mineral phases; thereby potentially simulating the behavior of admixtures. Specific details on the behavior of admixtures in this regard are not readily available.

The defined conditions are used to hydrate the virtual microstructure in cycles of dissolution, diffusion and reaction that mimic the observed behavior of Portland cement. Empirical rules based on observed behavior govern the hydration behavior of the specific phases, and a random walk algorithm [13] determines how ions move through the porosity of the virtual microstructure. One of the inherent limitations of the VCCTL is that no information is provided to define the specific time associated with each computational cycle, thus limiting the ability to simulate the time domain of the model [21]. By default, the elapsed hydration time is calculated according to equation 1 , where $\beta$ is a constant assigned based on empirical data, $\mathrm{t}$ is time, and $\mathrm{n}$ is the number of cycles.

$$
t=\beta \times n^{2}(1)
$$


When simulation of early age properties is a priority, the timing of the computations can be calibrated using a continuous hydration kinetics curve, obtained either through measurement of chemical shrinkage or isothermal conduction calorimetry. At the conclusion of each simulation cycle, the closest match to the simulated heat or shrinkage value is determined in the supplied shrinkage or heat curve. A simple linear interpolation algorithm computes the exact time at which the simulated value would match the experimental, and the length of the current simulation cycle is adjusted to match. This functionality compensates for discrepancies between the isothermal or shrinkage test temperature and simulation temperature, as well as changes in the temperature of the microstructure as the simulation progresses. These compensations are performed on a per-cycle basis, using the activation energy specified by the user for portland cement [22].

As the hydration progresses, properties of the evolving microstructure are recorded for each cycle or at user specified intervals of time. Available information includes the volume fractions of the different phases present over time, the heat released, the temperature of the simulated binder, and others. For this research, the most important property of the microstructure over time is the volume fraction of interconnected solids. The value for the fraction of solids connected is computed using a percolation algorithm $[23,24]$ and quantifies the transition of a Portland cement paste from a viscous suspension of particles to a rigid interconnected matrix, which is the fundamental description of the phenomenon of "set". This output is computed at user specified intervals by evaluating the percolation of solids through the simulated microstructure. Several studies [25-27,12] have examined the relationship between the simulated solids percolation results and experimental setting time via Vicat needle, generally for the purpose of experimental validation of the model. The time calibration functionality of the VCCTL has been utilized in a different study of methods for the detection of set [3], where a chemical shrinkage curve was used to calibrate the time scale of the model. It was found that the initial setting time via Vicat needle coincided with a dramatic increase in the fraction of interconnected solids.

Because the effects of admixtures on hydration manifest in the heat evolution of Portland cement, and heat of hydration data can be obtained simply through isothermal calorimetry, it was postulated that the use of heat of hydration curves obtained through calorimetry of different 
combinations of cement and admixture may enable the simulation of the influence of the admixtures on the setting time of Portland cement concrete. It is important to note that the assumption underlying this postulate is that admixtures influence the chemical reactivity of all portland cement components to the same degree. This is known to be false; however, it is also known that the bulk of the cement paste (the $\mathrm{C}_{3} \mathrm{~S}$ phase) is responsible for most of the heat evolution observed during hydration and initial setting. It may be possible to simulate admixtures that primarily act by hindering or accelerating the bulk phase in an acceptable manner.

\section{Experiment}

\subsection{Admixture Selection}

Commercially available and ASTM C494 [28] compliant admixtures were selected for this study, and included a lignosulfonate based type D water reducer and retarder, a polyacrylate based type $\mathrm{F}$ high range water reducer, a calcium chloride type E accelerator and water reducer, as well as a calcium nitrite based type $\mathrm{C}$ accelerator, which also meets the ASTM C1582 [29] standard for corrosion inhibiting admixtures. The selection of admixtures for this study was intentionally broad; all four have different classes of active chemical species and different intended usages. Low, moderate, and high dosage rates were selected for each admixture based on the manufacturer's recommended dosage range.

\subsection{Isothermal Conduction Calorimetry}

Isothermal conduction calorimetry was used to obtain heat of hydration curves for each admixture-dosage combination. The procedures for specimen preparation outlined in ASTM C1702 were followed. The total specimen mass was calculated to be approximately $6 \mathrm{~g}$ to facilitate optimum internal mixing. Admixtures were incorporated by preparation of a dilute solution for each dosage rate. Special care was taken to ensure precise thermal mass balance between specimen and reference vials, with the masses of the vials themselves accounted for in addition to the mass of specimen. Samples were allowed to reach thermal equilibrium overnight before the addition of the water-admixture solution, after which heat evolution was logged for 48 hours. 


\subsection{Time of Set Testing}

For each admixture type and dosage, a mortar was prepared and tested according to ASTM C 403. The use of the Vicat needle apparatus [30] for time of set testing was initially considered; however, this test is generally used on plain portland cement paste for the purpose of ensuring compliance with material specifications [14], not to determine the setting time of concrete. Additionally, the requirement that the paste have a specific "normal" consistency[31] was incompatible with the viscosity modifying effects of certain admixtures and the water to cement ratio chosen for the study. All mortar prepared for this research had the same ratio of cement to sand, which was chosen to be representative of the mortar that would be sieved from a concrete mix. Admixtures were premixed with the required quantity of water, to parallel the conditions of the isothermal calorimetry testing. The mortar was mixed using a stand mixer, and the initial temperature and ambient conditions during the test were recorded.

\subsection{Simulation Input and Parameters}

The cement compositional data used in this study was obtained via multiple methods. The volume fraction, surface area fraction, and two point spatial distribution functions required by the VCCTL were acquired as part of previous research, while XRD combined with rietveld refinement was used to obtain more comprehensive mass fraction data. The different compositional data used for this research is summarized in table 1. While quantitative X-ray diffraction allow for the acquisition of mass fractions for any crystalline phases present in the cement, the techniques obtaining volume and surface area fractions are tailored specifically to the clinker phases. 
Table 1: Cement Composition Data

\begin{tabular}{cccc}
\hline Phase & Mass \% (XRD) & Volume \% & Surface Area \% \\
\hline $\mathrm{C}_{3} \mathrm{~S}$ & 59.95 & 66.14 & 58.44 \\
$\mathrm{C}_{2} \mathrm{~S}$ & 13.67 & 15.72 & 20.37 \\
$\mathrm{C}_{4} \mathrm{AF}$ & 13.49 & 12.04 & 4.07 \\
$\mathrm{C}_{3} \mathrm{~A}-$ Cubic & 4.30 & 5.74 & 17.12 \\
$\mathrm{C}_{3} \mathrm{~A}-$ Orthorhombic & 0.59 & - & - \\
$\mathrm{CaCO}_{3}$ (Limestone) & 4.29 & - & - \\
$\mathrm{CaSO}_{4} * 2 \mathrm{H}_{2} \mathrm{O}$ (Gypsum) & 1.80 & - & - \\
$\mathrm{CaSO}_{4} * 0_{5} \mathrm{H}_{2} \mathrm{O}$ (Hemihydrate) & 0.70 & - & - \\
$\mathrm{CaSO}_{4}$ (Anhydrite) & 0.16 & - & - \\
\hline
\end{tabular}

The cement used for this study contained approximately $4.3 \%$ limestone. The limestone fraction as well as the mass fractions of the different sulfate phases via XRD (Table 3) were incorporated into all microstructures generated for this study. The particle size distribution for the cement to be simulated is also required as input to the VCCTL, and was measured via laser particle size analysis (figure 1).

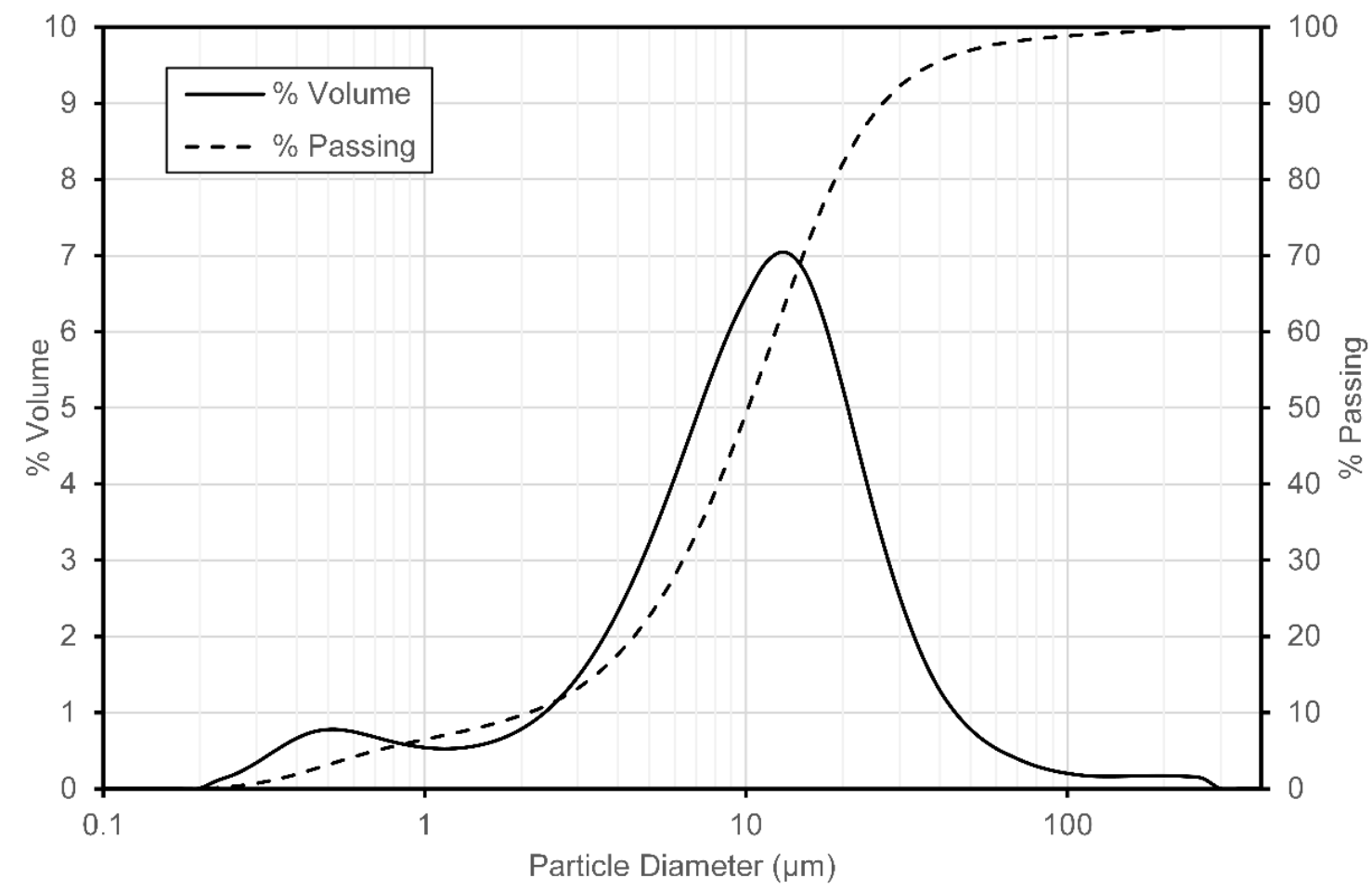

Fig 1 Volume and Cumulative particle size distributions used as input to the VCCTL 
The primary goal when choosing the initial state and environmental conditions for the model was fidelity to the corresponding experiments. The generation of the initial microstructure requires several inputs that are intended to simulate surface specific phenomena. The degree of dispersion setting in the VCCTL allows for the specification of the minimum number of pixels of water between each particle, with options for zero, one, and two pixels available. For mixtures with no water reducer, the dispersion was set to zero pixels. Dispersion distances for the lignosulfonate mid-range and polycarboxylate high-range water reducers were set to 1 and 2 pixels respectively, to mimic differences in their relative dispersive power, based on Björnström and Chandra [32]. Realistic particle shape data from the built-in VCCTL library was used in the generation of all microstructures. The resolution and size of the virtual microstructures were left unchanged, based on previous research [33].

During initial trial testing for time of set, it was noted that a significant amount of heat was released as the test neared final set, to the point where the specimen was warm to the touch relative to ambient conditions. Because time of setting tends to decrease with higher temperatures $[34,35]$, the semi-adiabatic thermal condition was chosen for simulating the time of set in this study. This allows for the specification of a heat transfer coefficient out of the virtual microstructure, which combined with the initial binder temperature and the temperature of the ambient environment enables the approximate simulation of any temperature rise. This technique is limited in that the actual thermal environment of the test specimen is more complex than a single, constant heat transfer coefficient. To determine the optimal temperature coefficient, the temperature of a control specimen was logged for 12 hours. For simulations of the mortar used in the study, priority was placed on matching the experimental temperature curve during the period up to final set. The resultant heat transfer coefficient chosen for simulations was $0.4 \mathrm{~W} / \mathrm{K}$. This heat transfer coefficient was used for all simulations; all time of set testing was performed in the same controlled environment with the same equipment and procedures. The difference in temperature between the test specimen and simulation using this heat transfer coefficient was less than $0.5{ }^{\circ} \mathrm{C}$ for the first 5 hours, after which the model temperatures began to exceed the measured values. This deviation was not unexpected; it is unlikely that the thermal conditions of a setting time specimen can be fully captured using a single heat transfer coefficient; this method is more realistic, however, than simply assuming the conditions of the test specimen are isothermal. For each hydration simulation, the temperature was set to the initial recorded 
temperature of the paste during the time of set test, the thermal state was set to isothermal, and the curing conditions were saturated. The specific heat of hydration curve for each admixture type and dosage was used to calibrate the time scale of the model. 


\section{Isothermal Conduction Calorimetry}

The power and total heat evolution curves obtained via conduction calorimetry and used as inputs for the VCCTL are presented in the following figures. The figures presented in the following pages observe certain conventions; all are presented with the control specimen as obtained from a plain portland cement at $0.45 \mathrm{w} / \mathrm{c}$, with a test temperature of $23^{\circ} \mathrm{C}$.

\subsection{Type D Admixture}
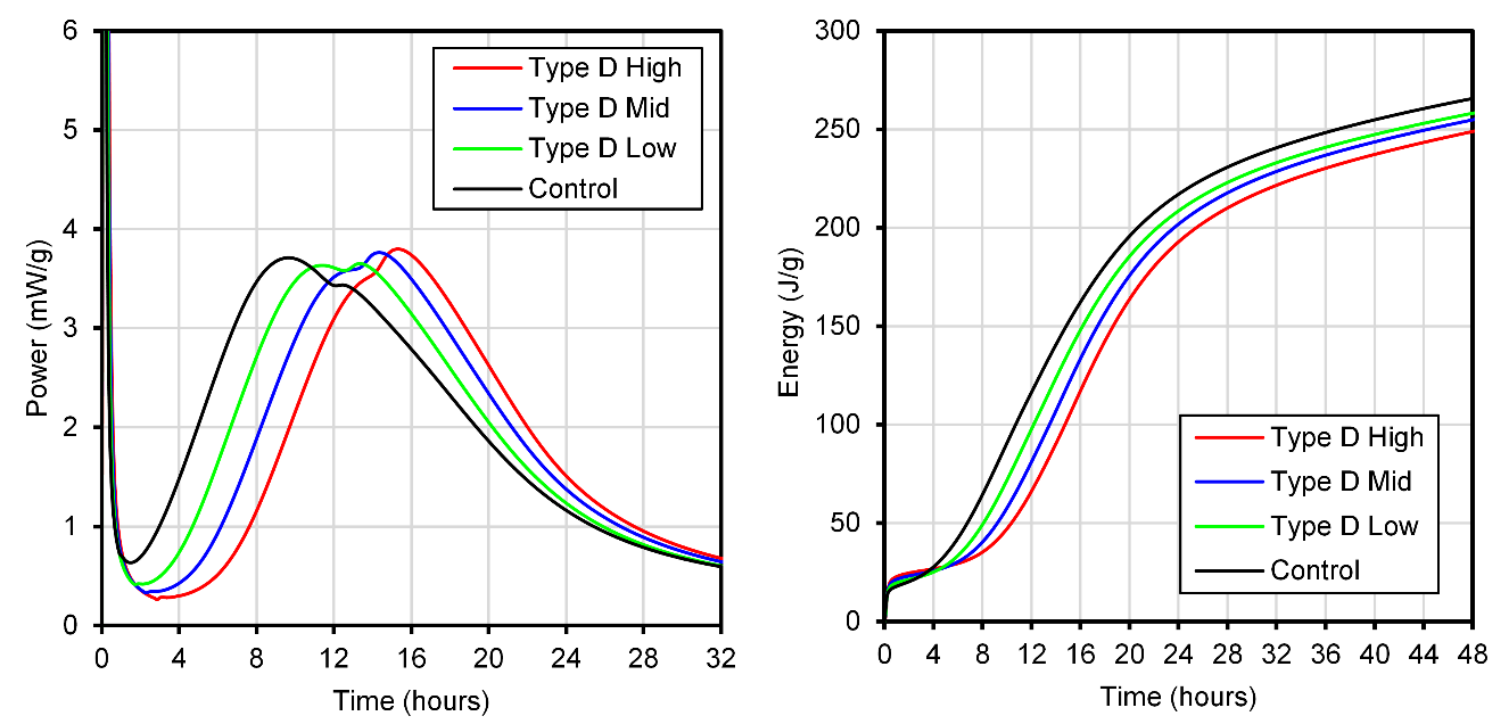

Fig 2 Power and energy curves normalized by cement mass for increasing dosages of type D admixture, with retardation of heat release occurring later at successively increasing dosages

The results from calorimetry of the type $\mathrm{D}$ admixtures are presented in figure 2 . The type D admixture exhibits significant retardation, with a delay of approximately two additional hours from the control at each increasing dose. The power curve in figure 1 also shows a shift in the prominence of the first and second peaks in the bulk hydration phase. The first peak is higher than the secondary for the control curve, and the first peak falls slightly and the second peak rises as the dosage rate increases. It is possible that this corresponds to enhanced aluminate reactivity, the commonly attributed source of the second peak during the bulk hydration phase [7]. The total energy curves for each dosage reflect the successive delay present in the power curves; the slopes of the total heat curves appear to be unchanged from the control after the conclusion of the beginning of the bulk hydration phase. 


\subsection{Type F Admixture}
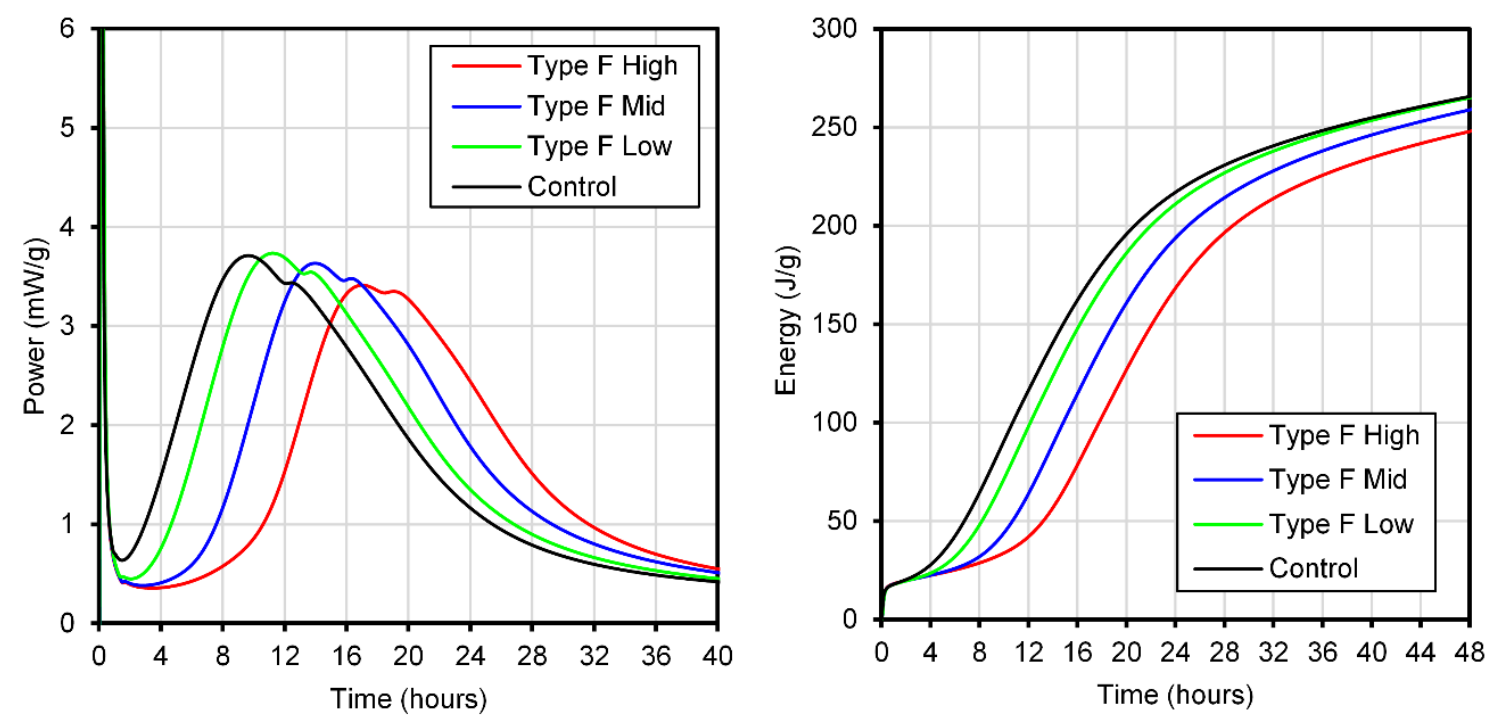

Fig 3 Type F admixture power and energy curves normalized by cement mass, with each increase in dosage resulting in a larger delay in heat release

The type $\mathrm{F}$ admixture induced a high degree of retardation (figure 3), with a slight retardation from the control at the lowest dosage, and approximately three additional hours of retardation with each successive dose. The retardation of heat release at the highest dosage rate is such that peak heat does not occur until approximately 17 hours, which is roughly 2 hours later than the highest dosage of the type D admixture. The relative prominences of the first and second peaks in the bulk hydration phase exhibit less of a shift than with the type D admixture, with the first peak falling slightly relative to the second with each dosage increase. The peak power generation also falls slightly with the moderate and high dosages of the type F admixture. The energy curves exhibit a steeper slope at the 48 hour mark when the type F admixture is present. This effect is pronounced enough that the lowest dosage has approximately the same 48 hour energy as the control, despite an initial delay in heat generation. This may be attributable to increased uniformity in the microstructure, resulting in more thorough hydration due to the dispersion of the cement particles. 


\subsection{Type E Admixture}
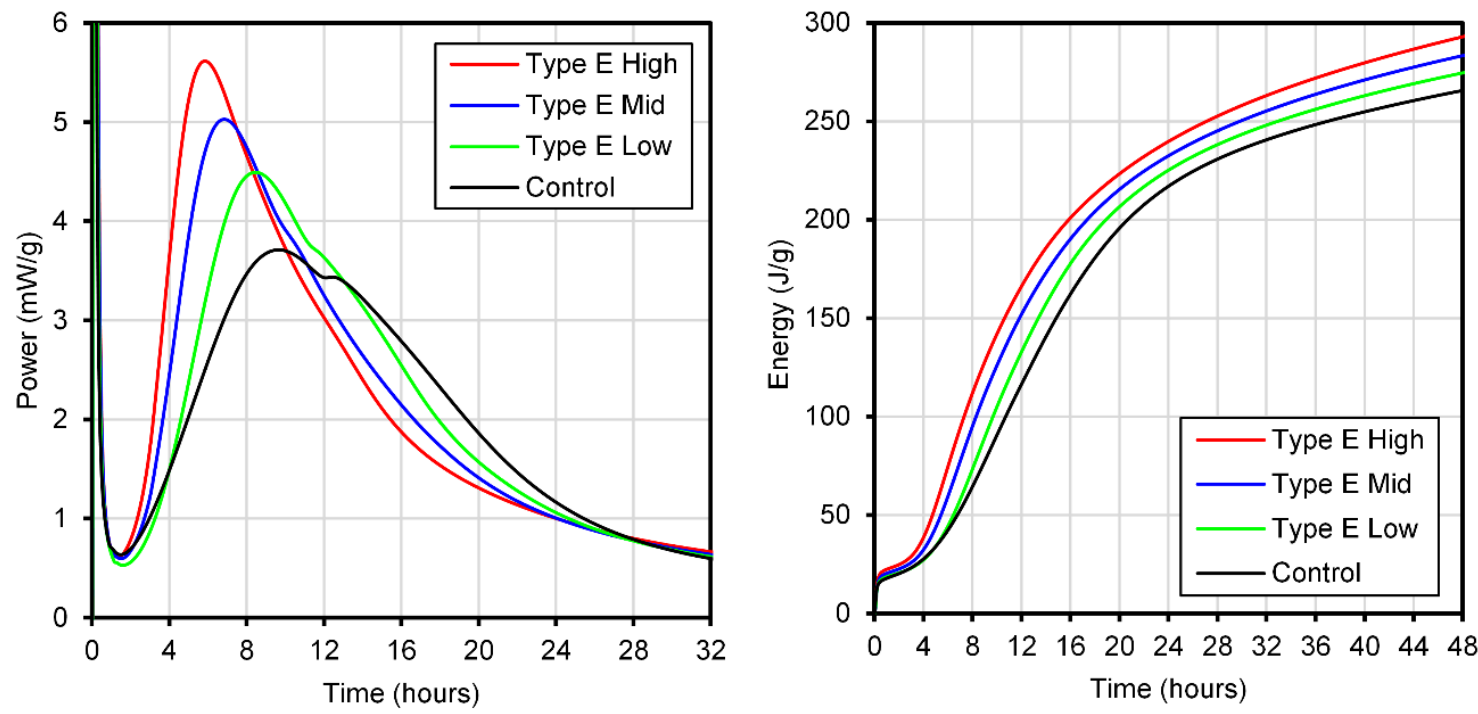

Fig 4 Power and energy curves normalized by cement mass for a Type E admixture, with peak power increasing in magnitude and occurring earlier with increasing dosage

The power and energy curves obtained from the type E accelerating admixture (figure 4) exhibit sharp contrast to those of the water reducing and retarding admixtures. While the type D and type $\mathrm{F}$ admixtures show slight changes in the shape of the bulk hydration peaks, their primary effect was that of a shift; the ultimate changes in the shapes of the peaks and the peak heat output were slight in comparison. The type E admixture exhibits substantial shape alteration of the bulk hydration power curve. The height and time of the first peak shifts up and to the left with each successive dosage increase; the highest dosage reaches a peak heat value approximately $50 \%$ higher and 4 hours earlier than the control. The secondary peak associated with enhanced aluminate reactivity, however, the shift is less substantial. The relative height of this second peak is slightly diminished as the dosage increases. The magnitude of the primary peak does make the secondary peak difficult to distinguish at high dosage rates; however, it is still present and occurs at approximately 13 hours.

The energy curves exhibit a less pronounced change in shape in comparison to the power curves for the type E admixture; they do show the accelerating effect of increased dosages, and there is a gradual change in the curvature to a more rounded shape. The slope of the energy curves at 48 hours also increases slightly at the higher dosage rates. Type E admixtures are both 
accelerators and water reducers. It is possible this effect is again due to a more thorough hydration resulting from a more uniform microstructure caused by the dispersion of cement particles.

\subsection{Type C Admixture}
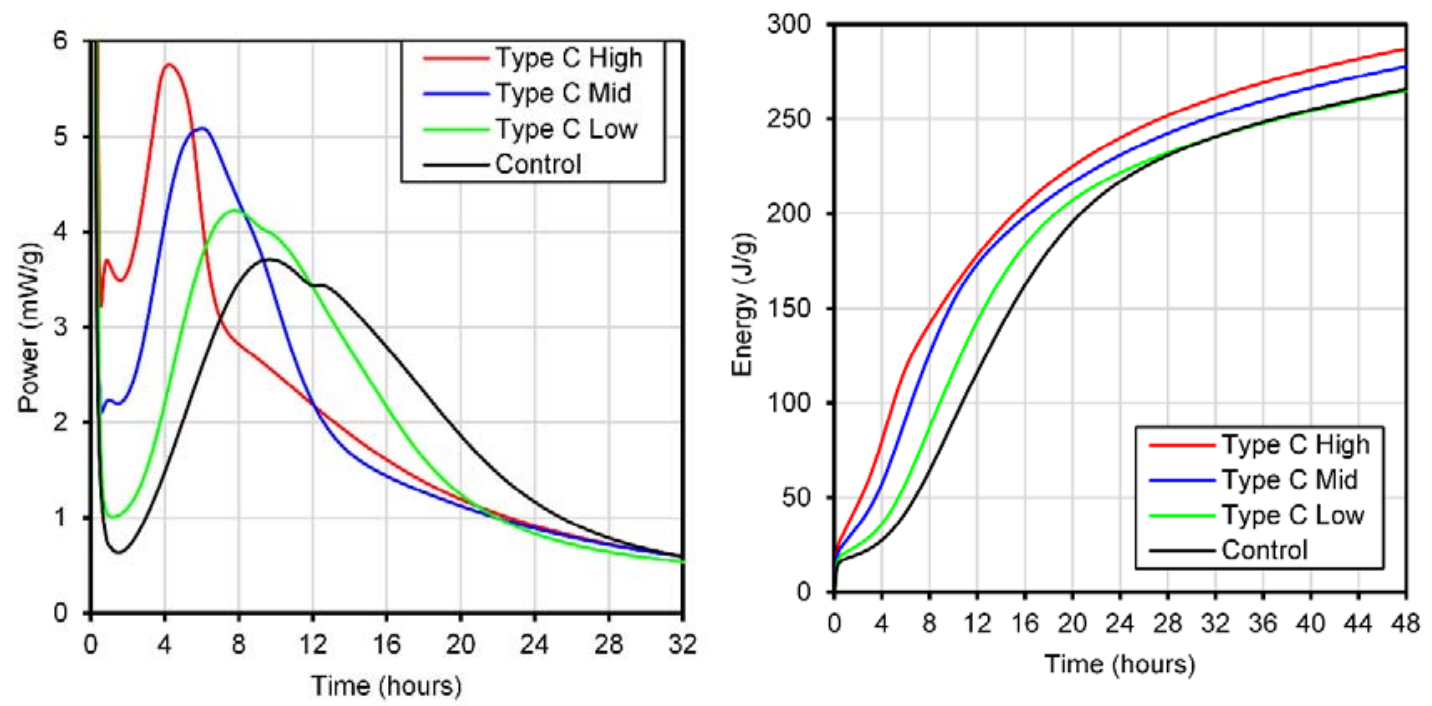

Fig 5 Type C/corrosion inhibiting admixture power and energy curves, with increasing dosage resulting in earlier heat release and higher heat release during the induction period

Power and energy curves for the different dosage of the type $\mathrm{C}$ admixture exhibit a dramatic change both in the time to reach peak values and the general shapes of the curves. Each successive increase in dosage results in an increase in peak height, decrease in time to peak, and an increase in the power generated by the reaction during the induction period. The highest dosage exhibits an induction phase power generation equal to that of peak power generated by the control. A small peak prior to the bulk phase also appears immediately after the initial peak that corresponds to initial dissolution of $\mathrm{C}_{3} \mathrm{~A}$ and mixing. The type $\mathrm{C}$ admixture used in this study was calcium nitrite based; prior work has attributed the increase in peak height to increased ettringite formation [36] in admixtures of this type. The energy curves reflect the dramatic alteration of the early power curves, with gradually more substantial change with increases in dosage. 


\section{Comparison of Set Predictions to Experimental Measurements}

Each of the energy curves obtained from the admixture-dosage combinations discussed in section three were used as inputs for hydration simulations. The same combinations were prepared as mortars and tested adhering to the procedure defined in ASTM C 403. Comparison of the simulation results with the measured penetration resistance values requires the definition of equivalent values to provide consistent comparisons for all combinations. These equivalent values were defined using the control simulations and time of set test.

\subsection{Control Specimen}

Both the fraction solids connected value computed by the VCCTL and the penetration resistance measurements obtained per ASTM C403 provide methods to quantify the degree to which the cementitous matrix has become rigid. They do not, however, provide the same type of measurements. Penetration resistance is a measurement of the yield stress of the hydrating material, while the solids connected value provides a direct quantification of the phenomena underlying the behavior of set. These measurements are not directly comparable without some type of calibration. Because the potential accuracy of the VCCTL with respect to admixture simulation is being examined by this study, a calibration of the two types of measurements was developed using the controls.

Figure 6 exhibits the penetration resistance development of the mortar on the left axis, with the corresponding values of initial and final set denoted by thin black horizontal lines at the standard locations. The right axis corresponds to the solids connected values for the two simulation trials. The scale for the vertical axis is set such that every $3.445 \mathrm{MPa}(500)$ psi of penetration resistance corresponds to a $10 \%$ increase in solids connected. This results in the crossover points of the penetration resistance curves and the simulated solids connected values occurring concurrently with initial and final set. Extrapolating from this, the solids connected values that correspond to initial and final set are 0.1 and 0.8 , respectively. 


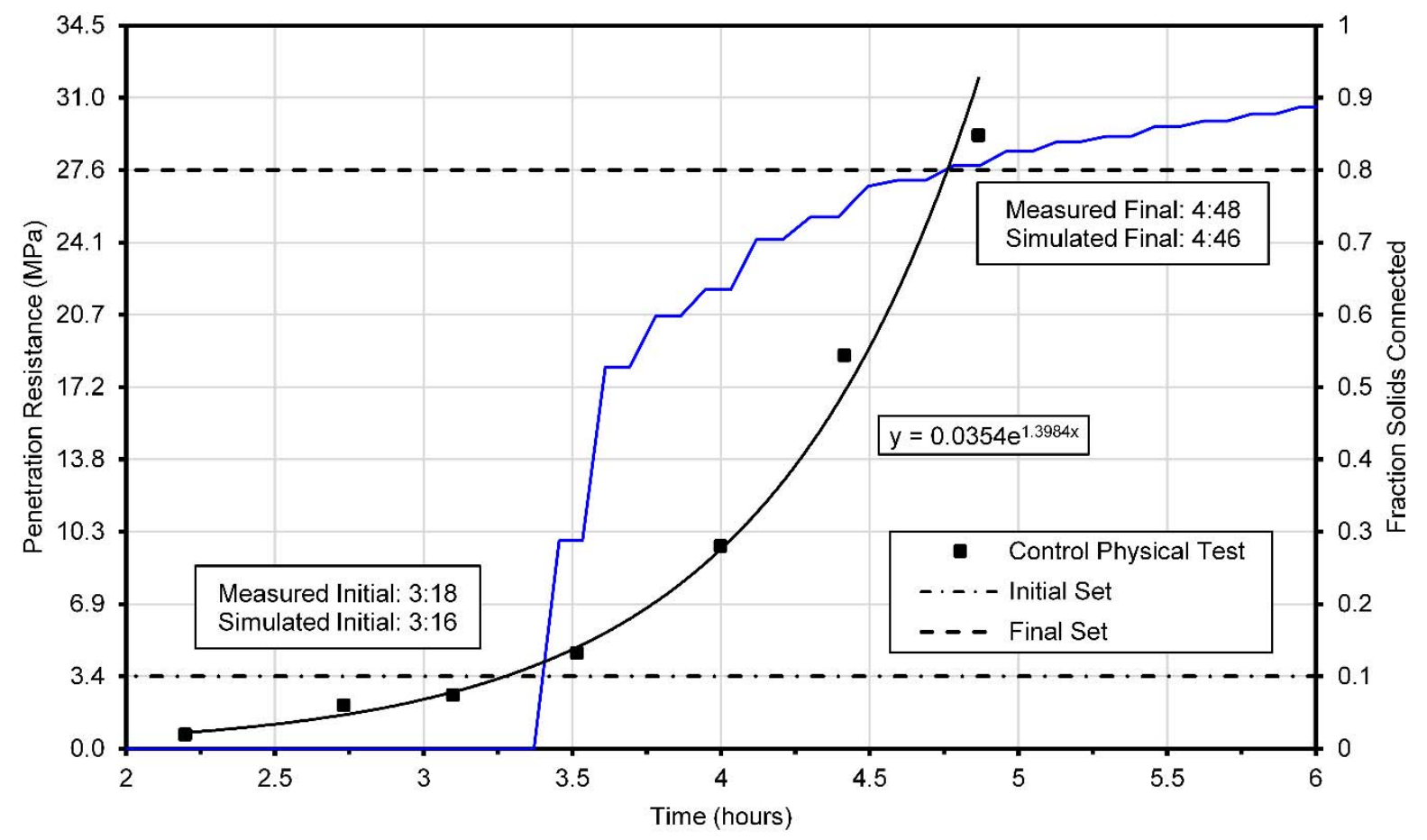

Fig 6 Comparison of simulated (blue) and measured setting times for the control specimen

Regardless of the scale, the development of strength and solids connected occurs within the same 1 hour window. The equivalent values developed using the controls were used to gauge the effectiveness of all the admixture-dosage combinations examined in this study. 


\subsection{Type D Admixture}

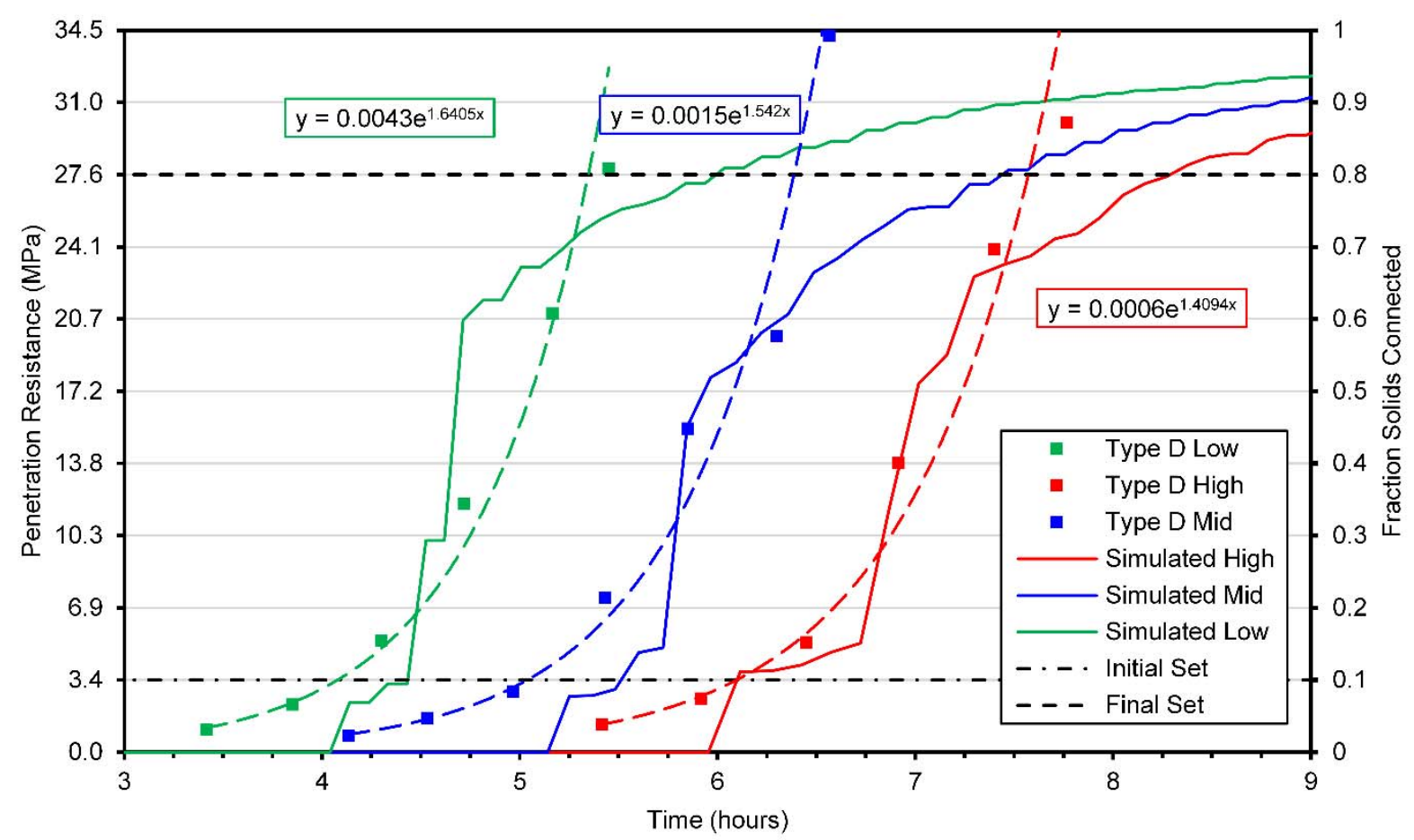

Fig 7 Comparison of simulated and measured set results for increasing dosages of type D admixture

Measured initial set times for the type D specimens (figure 7) ranged from 4 hours to 6 hours, while measured final set times ranged from 5.5 to 7.5 hours. The time from initial to final set was approximately 1.5 hours for all dosage rates. The simulated set times generally overestimated time of set by one hour or less. There was no discernable change in the degree of overestimation as dosage increased. 


\subsection{Type F Admixture}

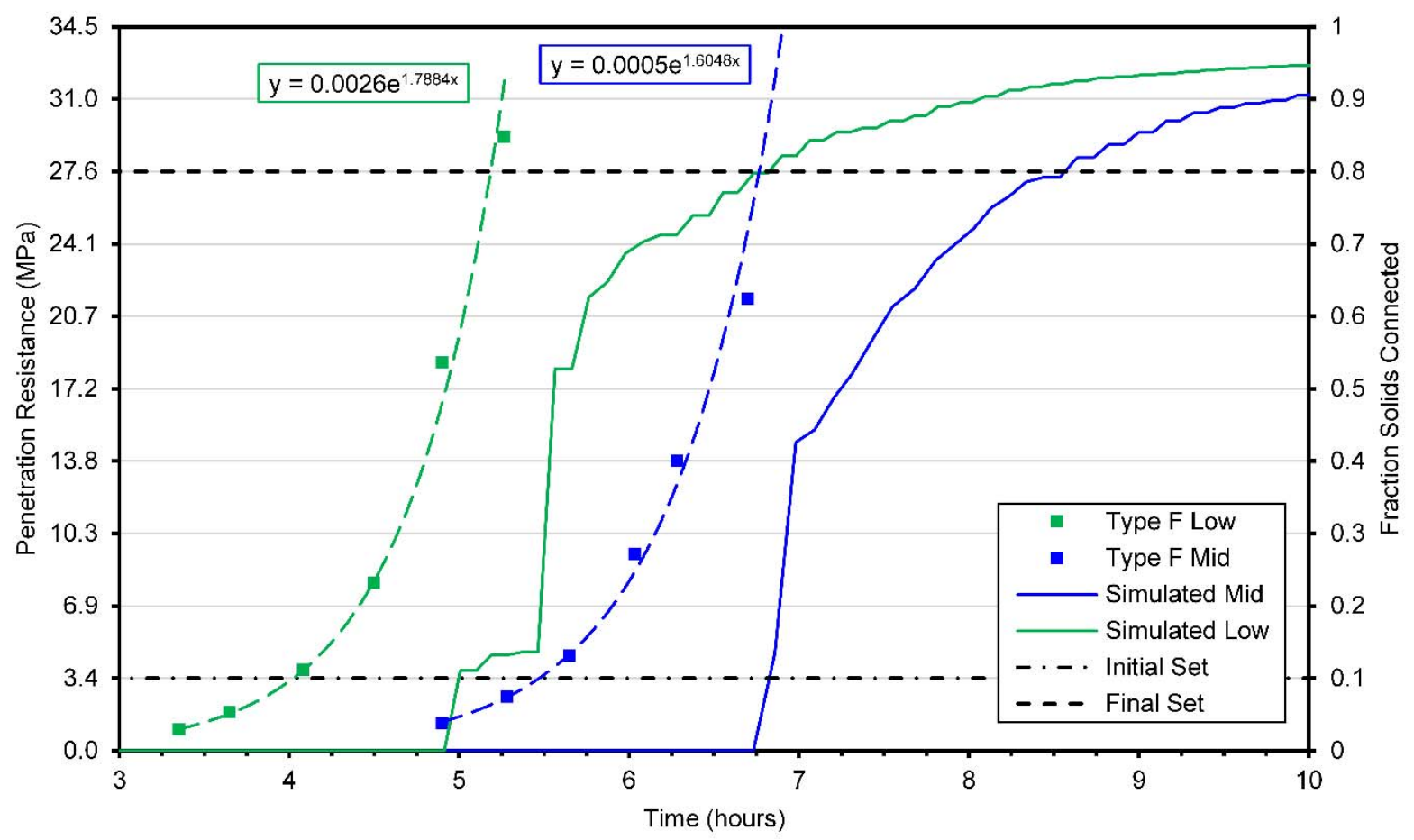

Fig 8 Comparison of simulated and measured set results for increasing dosages of type F admixture, with simulations predicting later set than measured

The specimens containing Type F admixtures (figure 8) reached initial set at approximately 4 hours for the lowest dose, and 5.5 hours for the moderate dose. Final set occurred just over one hour after initial set in both cases. A larger discrepancy between simulated and measured set times resulted with the type F predictions, with an overestimation of more than one hour for initial set and nearly two hours for final set. As with the type D admixture, no trend in degree of overestimation was observed with admixture dosage. The highest dosage of type $\mathrm{F}$ admixture is notably absent from the results; the combination of the relatively high water to cement ratio of 0.45 and a maximum dosage of type $\mathrm{F}$ admixture resulted in a mixture with excessive segregation, rendering the sample unsuitable for penetration resistance measurements. 


\subsection{Type E Admixtures}

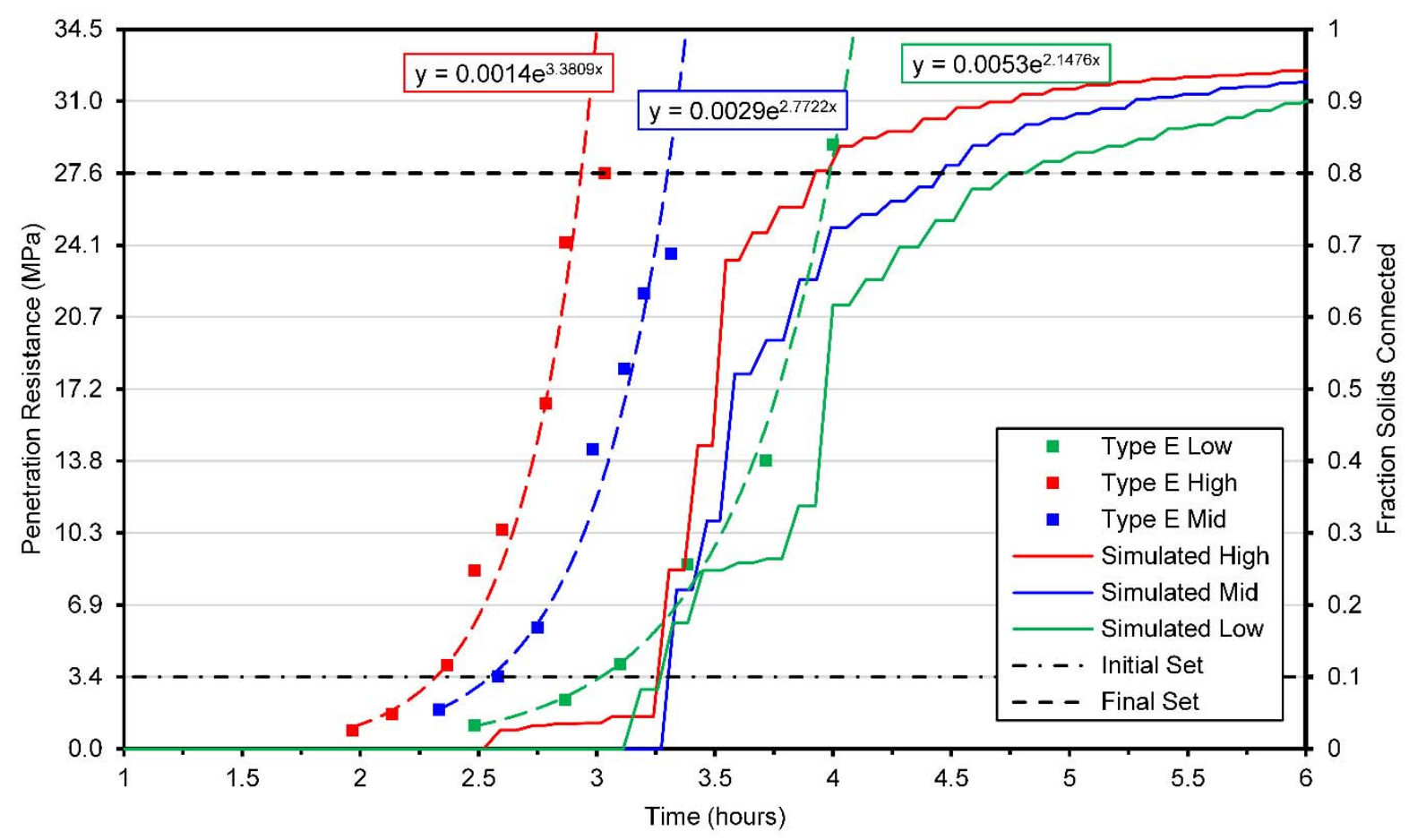

Fig 9 Comparison of simulated and measured set results for increasing dosages of type $\mathrm{E}$ admixture

Measured initial set times for the specimens containing Type E admixture (figure 9) decreased with increasing dosage, and ranged between 3 hours and 2.25 hours, while final set occurred between 1 hour and 0.5 hours after initial. The development of penetration resistance was more rapid as the admixtures dosage increased. Simulated initial and final set occurred later than measured results for all dosages, with an increase in error as dosage rose. The simulated predictions for initial setting time remained relatively constant at each dosage, while the predicted final setting times shifted from approximately 5 hours at the lowest dosage, down to 4 hours at the highest dosage. 


\subsection{Type C Admixture}

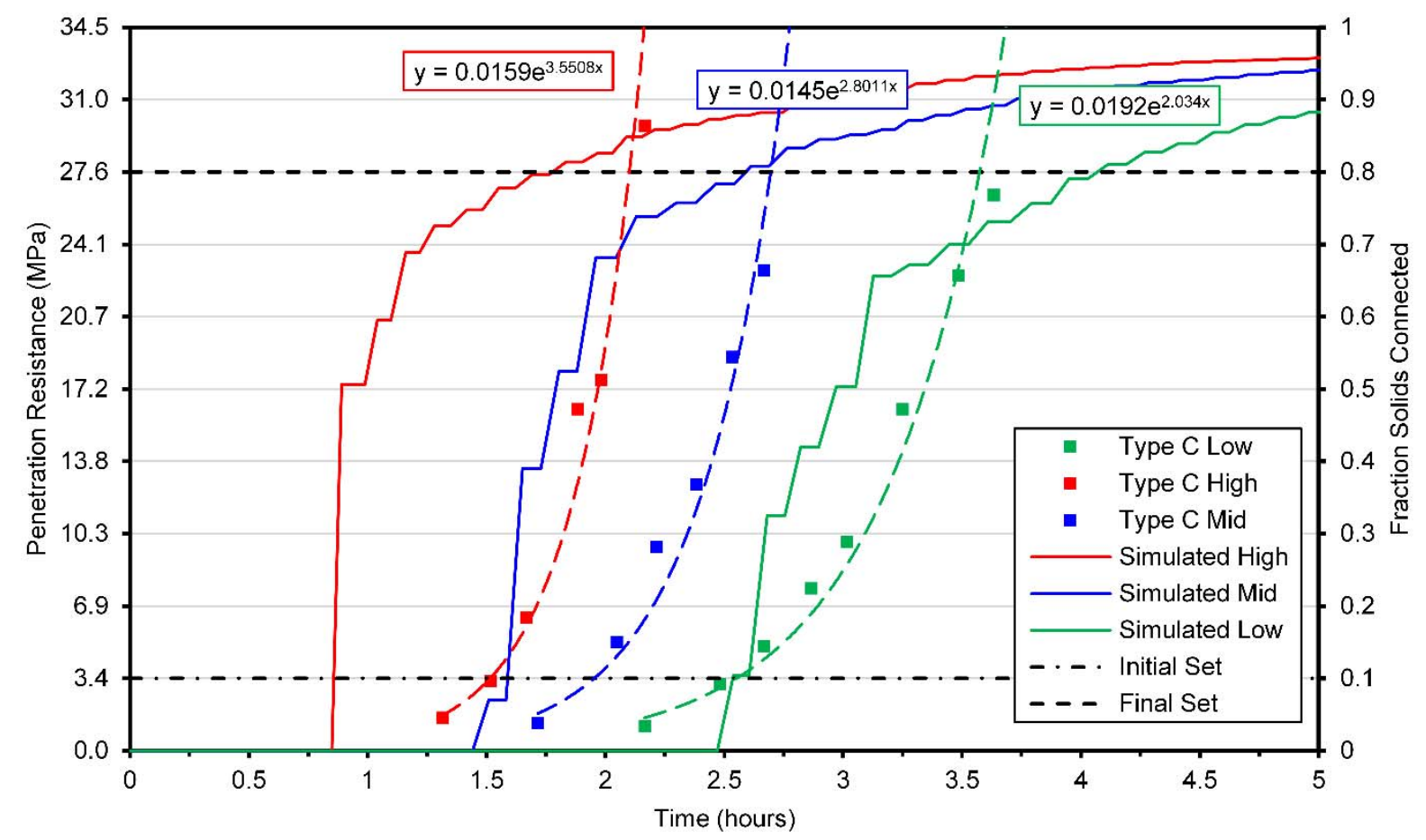

Fig 10 Comparison of simulated and measured set results for increasing dosages of type C admixture

Initial set for the type $\mathrm{C}$ specimens (figure 10) occurred at approximately 2.5 hours for the low dosage, 2 hours for the moderate dosage, and 1.5 hours for the high dosage. For both simulated and measured values of set time, increasing dosage resulted in faster set times, though this effect is more exaggerated in the simulated results. The time required to reach final set after initial set occurs is overestimated by the model; this combined with general overestimation of the effect of increasing dosage results in good initial set agreement for the low dosage, decent final set agreement for the moderate dosage, and fair final set agreement at the highest dosage. 


\section{Discussion and Conclusions}

Table 2: Summary of Measured and Simulated Set Times

\begin{tabular}{r|c|ccc|cc|ccc|ccc} 
Admixture & & Type D & & & Type F & & Type E & & Type C & Mid & High \\
\hline Dosage & Control & Low & Mid & High & Low & Mid & Low & Mid & High & Low & Mid & \\
\hline $\begin{array}{r}\text { Measured } \\
\text { Initial }\end{array}$ & $03: 18$ & $04: 03$ & $05: 03$ & $06: 07$ & $04: 00$ & $05: 28$ & $03: 01$ & $02: 35$ & $02: 19$ & $02: 30$ & $01: 56$ & $01: 31$ \\
$\begin{array}{r}\text { Simulated } \\
\text { Initial }\end{array}$ & $03: 16$ & $04: 25$ & $05: 30$ & $06: 05$ & $04: 59$ & $06: 49$ & $03: 16$ & $03: 18$ & $03: 15$ & $02: 32$ & $01: 35$ & $00: 51$ \\
$\begin{array}{r}\text { Measured } \\
\text { Final }\end{array}$ & $04: 48$ & $05: 26$ & $06: 27$ & $07: 37$ & $05: 13$ & $06: 48$ & $03: 58$ & $03: 21$ & $03: 02$ & $03: 39$ & $02: 44$ & $02: 08$ \\
$\begin{array}{r}\text { Simulated } \\
\text { Final }\end{array}$ & $04: 46$ & $05: 59$ & $07: 26$ & $08: 17$ & $06: 49$ & $08: 33$ & $04: 44$ & $04: 27$ & $03: 55$ & $04: 04$ & $02: 35$ & $01: 46$ \\
\hline $\begin{array}{r}\text { Initial Set } \\
\text { Error (m) } \\
\text { Final Set }\end{array}$ & $00: 02$ & $00: 22$ & $00: 27$ & $00: 02$ & $00: 59$ & $01: 21$ & $00: 15$ & $00: 43$ & $00: 56$ & $00: 02$ & $00: 21$ & $00: 40$ \\
Error (m) & $00: 01$ & $00: 33$ & $00: 59$ & $00: 40$ & $01: 36$ & $01: 45$ & $00: 46$ & $01: 06$ & $00: 53$ & $00: 25$ & $00: 09$ & $00: 22$
\end{tabular}

The addition of early age heat of hydration data to VCCTL produces very accurate results when predicting the set time for unmodified mortar. The results of the initial and final set predictions for the control specimen agree with the measured results via penetration resistance to within less than 10 minutes. A convenient metric for evaluation of the viability of the predicted set times can be found within ASTM C403. This standard specifies maximum multi-operator precision values for initial and final set of 19.4 minutes and 28.7 minutes respectively; the simulated set time values for the control are within these limits. The accuracy of the results for the control specimen supports the methodology of maximum realism when selecting parameters for simulations.

For certain types of admixtures, the incorporation of heat of hydration data into the VCCTL allows for useful prediction of time of set. Simulations for type D water reducer were higher than the multi-operator precision values for initial setting at low and moderate dosages, and within two minutes at the highest dose. The final setting projections for the low dosage was also higher than the values specified in ASTM C 403. For type C admixtures, predictions for all dosage rates were within multi-operator precision with the exception of initial set at the moderate and high dosages.

The type F admixture used in this study exhibited the largest discrepancies between the predicted and measured values. This is attributed to physiochemical nature of polycarboxylate modified cement paste in a low shear, internally mixed isothermal calorimetry cell. It has been found that the retardation seen in the power curves from paste is not evident in the behavior of 
concrete containing these admixtures [37], where shear stresses during mixing are greater. This highlights a limitation of utilizing isothermal calorimetry exclusively for the prediction of setting time. It is possible that similar effects are responsible for the poor predictions of setting for the type $\mathrm{C}$ admixture, however further investigation is required.

Generally speaking, the accuracy for predictions of mixtures containing admixtures decreased as the dosage of the respective admixture increased. The deviation of the predictions from reality with increasing dosage is a reflection of the nature of the hydration simulation. Supplying a characteristic heat of hydration curve for each admixture does compensate for the resulting change in setting time; however, the simulations do not take into account any possible changes to the relative reaction rates of the hydrating phases, or changes in the properties of the resulting hydration products. As dosage increases, the chemical discrepancies between the behavior of the virtual and physical hydrations become more extreme. It is likely that the thermal behavior of admixtures in concrete is only part of the information required to accurately simulate early age properties.

Several conclusions can be drawn directly from the results of this study:

1. The VCCTL can be used to accurately predict the time of set in mortar incorporating admixtures based on the techniques developed herein.

2. Predictions for type $\mathrm{D}$ and type $\mathrm{C}$ admixtures fall within the precision statements specified by ASTM C 403 in certain cases, and are otherwise accurate to within one hour. In no instances are the predicted set times made less accurate by the incorporation of characteristic heat of hydration data.

3. The VCCTL predicts initial and final set of plain portland cement mortar within an accuracy of 5 minutes when calibrated with a heat of hydration curve.

4. Setting tests that are preformed using standardized equipment in consistent environmental conditions, the temperature rise in mortar can be accurately approximated by the VCCTL using the semi-adiabatic thermal condition.

5. Predictions using the technique presented herein did not produce accurate results for mortar mixtures which contain type F water reducing admixtures. This can be attributed to the behavior of polycarboxylate-modified cement paste in the low shear environment of an internally mixed isothermal calorimeter cell. 


\section{Acknowledgements}

The author would like to acknowledge the Florida Department of Transportation for funding this research under contract BDK75-977-73, as well as Jeffrey Bullard at the National Institute of Standards and Technology, for helpful discussion regarding the implementation of this research and insightful comments on this manuscript. 


\section{References}

[1] S. Mindess, F. Young, D. Darwin, Concrete, Prentice-Hall, Inc, Upper Saddle River, NJ, 2003.

[2] A. Neville, Properties of Concrete, 5th ed., Prentice Hall, San Francisco, CA, 2011.

[3] G. Sant, M. Dehadrai, D. Bentz, P. Lura, C.F. Ferraris, J.W. Bullard, et al., Detecting the Fluid-to-Solid Transition in Cement Pastes, Concr. Int. 31 (2009) 53-58.

[4] H. Taylor, Cement Chemistry, 2nd ed., Thomas Telford Publishing, London, 2004.

[5] J.W. Bullard, H.M. Jennings, R.A. Livingston, A. Nonat, G.W. Scherer, J.S. Schweitzer, et al., Mechanisms of cement hydration, Cem. Concr. Res. 41 (2011) 1208-1223. doi:10.1016/j.cemconres.2010.09.011.

[6] P. Hewlett, Lea's Chemistry of Cement and Concrete, 4th ed., Elsevier Ltd, 2006.

[7] K. De Weerdt, Combining Plasticizers/Retarders and Accelerators, Katholieke Universiteit Leuven, 2006.

[8] J. Cheung, A. Jeknavorian, L. Roberts, D. Silva, Impact of admixtures on the hydration kinetics of Portland cement, Cem. Concr. Res. 41 (2011) 1289-1309. doi:10.1016/j.cemconres.2011.03.005.

[9] K. Yamada, T. Takahashi, S. Hanehara, M. Matsuhisa, Effects of the Chemical Structure on the Properties of Polycarobxylate-type Superplasticiser, Cem. Concr. Res. 30 (2000) 197207. doi:10.1016/S0008-8846(99)00230-6.

[10] V.K. Peterson, M.C.G. Juenger, Time-resolved quasielastic neutron scattering study of the hydration of tricalcium silicate: Effects of $\mathrm{CaCl} 2$ and sucrose, Phys. B Condens. Matter. 385-386, Part 1 (2006) 222-224. doi:10.1016/j.physb.2006.05.194.

[11] ASTM C1702, Standard Test Method for Measurement of Heat of Hydration of Hydraulic Cementitious Materials Using Isothermal Conduction Calorimetry, ASTM International, West Conshohocken, PA, 2014. http://www.astm.org/Standards/C1702.htm (accessed August 23, 2014).

[12] J.W. Bullard, P.E. Stutzman, Analysis of CCRL proficiency cements 151 and 152 using the Virtual Cement and Concrete Testing Laboratory, Cem. Concr. Res. 36 (2006) 1548-1555. doi:10.1016/j.cemconres.2006.05.024.

[13] D.P. Bentz, Three-Dimensional Computer Simulation of Portland Cement Hydration and Microstructure Development, J. Am. Ceram. Soc. 80 (1997) 3-21. doi:10.1111/j.11512916.1997.tb02785.x.

[14] ASTM C150, Standard Specification for Portland Cement, ASTM International, West Conshohocken, PA, 2012. 
[15] R.D. Hooton, M. Nokken, M.D.A. Thomas, Portland-Limestone Cement: State-of-the-Art Report and Gap Analysis for CSA A 3000, Cement Association of Canada, Ottawa, Ontario, 2007.

[16] I. Soroka, N. Stern, Effect of Calcareous Fillers on Sulfate Resistance of Portland Cement, Am. Ceram. Soc. Bull. 55 (1976) 597-595.

[17] D.P. Bentz, Modeling the influence of limestone filler on cement hydration using CEMHYD3D, Cem. Concr. Compos. 28 (2006) 124-129. doi:10.1016/j.cemconcomp.2005.10.006.

[18] M. Collepardi, Admixtures Used to Enhance Placing Characteristics of Concrete, Cem. Concr. Compos. 20 (1998) 103-112.

[19] C. Jolicoeur, M.-A. Simard, Chemical Admixture-Cement Interactions: Phenomenology and Physico-chemical Concepts, Cem. Concr. Compos. 20 (1998) 87-101.

[20] J. Bullard, E.J. Garboczi, A model investigation of the influence of particle shape on portland cement hydration, Cem. Concr. Res. 36 (2006) 1007-1015. doi:10.1016/j.cemconres.2006.01.003.

[21] J.J. Thomas, J.J. Biernacki, J.W. Bullard, S. Bishnoi, J.S. Dolado, G.W. Scherer, et al., Modeling and Simulation of Cement Hydration Kinetics and Microstructure Development, Cem. Concr. Res. 41 (2011) 1257-1278. doi:10.1016/j.cemconres.2010.10.004.

[22] J.W. Bullard, D.P. Bentz, Disrealnew, National Institute of Standards and Technology, 2014.

[23] D.P. Bentz, E.J. Garboczi, Percolation of Phases in a Three-Dimensional Cement Paste Microstructural Model, Cem. Concr. Res. 21 (1991) 356-344.

[24] E.J. Garboczi, D.P. Bentz, N.S. Martys, Digital Images and Computer Modeling, in: Methods Phys. Porus Media, 1999: pp. 1-41.

[25] D.P. Bentz, C.J. Haecker, X.P. Feng, P.E. Stutzman, Prediction of Cement Physical Properties by Virtual Testing, in: Fifth Int. VDZ Congr. Proc., Dusseldorf, Germany, 2002: pp. 53-63.

[26] A. Princigallo, P. Lura, K. van Breugel, G. Levita, Early development of properties in a cement paste: A numerical and experimental study, Cem. Concr. Res. 33 (2003) 10131020. doi:10.1016/S0008-8846(03)00002-4.

[27] D.P. Bentz, E.J. Garboczi, J.W. Bullard, C.F. Ferraris, N.S. Martys, P.E. Stutzman, Virtual Testing of Cement and Concrete, in: J. Lamond, J. Pielert (Eds.), Significance Tests Prop. Concr. Concr.-Mak. Mater., ASTM International, 100 Barr Harbor Drive, PO Box C700, West Conshohocken, PA 19428-2959, 2006. http://www.astm.org/doiLink.cgi?STP169DEB (accessed November 18, 2015). 
[28] ASTM C494, Standard Specification for Chemical Admixtures for Concrete, ASTM International, West Conshohocken, PA, 2014. http://www.astm.org/Standards/C494.htm (accessed August 23, 2014).

[29] ASTM C1582, Standard Specification for Admixtures to Inhibit Chloride-Induced Corrosion of Reinforcing Steel in Concrete, ASTM International, West Conshohocken, PA, 2011. http://www.astm.org/Standards/C1582.htm (accessed August 23, 2014).

[30] ASTM C191, Standard Test Methods for Time of Setting of Hydraulic Cement by Vicat Needle, ASTM International, West Conshohocken, PA, 2013.

[31] ASTM C187, Standard Test Method for Amount of Water Required for Normal Consistency of Hydraulic Cement Paste, ASTM International, West Conshohocken, PA, 2011. http://compass.astm.org/EDIT/html_annot.cgi?C187+11e1 (accessed August 23, 2014).

[32] J. Björnström, S. Chandra, Effect of Superplasticizers on the Rheological Properties of Cements, Mater. Struct. 36 (2003) 685-692.

[33] E.J. Garboczi, D.P. Bentz, The Effect of Statistical Fluctuation, Finite Size Error, and Digital Resolution on the Phase Percolation and Transport Properties of the NIST Cement Hydration Model, Cem. Concr. Res. 31 (2001) 1501-1514.

[34] R.G. Burg, The Influence of Casting and Curing Temperature on the Properties of Fresh and Hardened Concrete, Portland Cement Association, Skokie, IL, 1996.

[35] S.H. Kosmatka, M.L. Wilson, Design and Control of Concrete Mixtures, Portland Cement Association, Skokie, IL, 2015.

[36] P. Sandberg, C. Porteneuve, F. Serafin, J. Boomer, N. LoConte, V. Gupta, et al., Effect of Admixture on Cement Hydration Kinetics by Synchrotron XRD and Isothermal Calorimetry, in: Int. Congr. Chem. Cem., 2008.

[37] S.A. Farrington, Evaluating the Effect of Mixing Method on Cement Hydration in the Prescence of a Polycarboxylate High-Range Water Reducing Admixture by Isothermal Calorimetry, in: Proc. Twelfth Int. Congr. Chem. Cem., Montreal, Canada, 2007. 